University of Wollongong

Research Online

Faculty of Engineering and Information

Faculty of Engineering and Information

Sciences - Papers: Part A

Sciences

$1-1-2014$

\title{
Novel algorithms for complete targets coverage in energy harvesting wireless sensor networks
}

Changlin Yang

University of Wollongong, cy116@uowmail.edu.au

Kwan-Wu Chin

University of Wollongong, kwanwu@uow.edu.au

Follow this and additional works at: https://ro.uow.edu.au/eispapers

Part of the Engineering Commons, and the Science and Technology Studies Commons

Research Online is the open access institutional repository for the University of Wollongong. For further information contact the UOW Library: research-pubs@uow.edu.au 


\title{
Novel algorithms for complete targets coverage in energy harvesting wireless sensor networks
}

\begin{abstract}
This paper addresses the problem of maximizing the network lifetime of rechargeable Wireless Sensor Networks (WSNs) whilst ensuring all targets are monitored continuously by at least one sensor node. The objective is to determine a group of sensor nodes, and their wake-up schedule such that within a time interval, one subset of nodes are active whilst others enter the sleep state to conserve energy as well as recharge their battery. We propose a Linear Programming (LP) based solution to determine the activation schedule of sensor nodes whilst affording them recharging opportunities and the same time ensures complete target coverage. The results show our LP solution achieves more than twice the performance in terms of network lifetime as compared to similar algorithms developed for finite battery WSNs. However, it is computationally expensive. We therefore propose Maximum Utility Algorithm (MUA), a few orders of magnitude faster approach that achieves $3 / 4$ of the network lifetime obtained by our LP solution.
\end{abstract}

\section{Keywords}

harvesting, energy, algorithms, complete, targets, wireless, coverage, sensor, networks, novel

\section{Disciplines}

Engineering | Science and Technology Studies

\section{Publication Details}

C. Yang and K. Chin, "Novel algorithms for complete targets coverage in energy harvesting wireless sensor networks," IEEE Communications Letters, vol. 18, (1) pp. 118-121, 2014. 


\title{
Novel Algorithms for Complete Targets Coverage in Energy Harvesting Wireless Sensor Networks
}

\author{
Changlin Yang and Kwan-Wu Chin *
}

\begin{abstract}
This paper addresses the problem of maximizing the network lifetime of rechargeable Wireless Sensor Networks (WSNs) whilst ensuring all targets are monitored continuously by at least one sensor node. The objective is to determine a group of sensor nodes, and their wake-up schedule such that within a time interval, one subset of nodes are active whilst others enter the sleep state to conserve energy as well as recharge their battery. We propose a Linear Programming (LP) based solution to determine the activation schedule of sensor nodes whilst affording them recharging opportunities and at the same time ensures complete target coverage. The results show our LP solution achieves more than twice the performance in terms of network lifetime as compared to similar algorithms developed for finite battery WSNs. However, it is computationally expensive. We therefore propose Maximum

*Authors Yang and Chin are with the School of Electrical, Computer, and Telecommunication Engineering, University of Wollongong, NSW, Australia, e-mail: cy116@uowmail.edu.au, kwanwu@uow.edu.au $\dagger$
\end{abstract}

Utility Algorithm (MUA), a few orders of magnitude faster approach that achieves $3 / 4$ of the network lifetime obtained by our LP solution.

\section{Introduction}

Wireless Sensor Networks (WSNs) are able to monitor their surroundings and route sensed data to one or more gateways via multi-hop communications wirelessly [1]. A fundamental problem in conventional WSNs is the finite battery lifetime of sensor nodes. A promising solution to this problem is to equip sensor nodes with the ability to harvest energy from its environment; e.g., solar [2]. Hence, assuming energy neutral operation [3], a WSN with said sensor nodes can operate perpetually.

In this paper, we consider the Maximum Lifetime Coverage (MLC) [1] problem in the context of rechargeable WSNs. The goal is to maximize a WSN's lifetime whilst ensuring all targets are monitored by at least one sensor node at all times. The authors of [4][5] and [6] propose to divide sensor nodes into set covers that collectively monitor all targets. An activation 
schedule is then computed for each cover such that the coverage lifetime is maximized. In particular, Liu et al. [5] present a Linear Program (LP) to maximize network lifetime subject to energy and connectivity constraints. Critically, these solutions do not consider recharging opportunities. To date, existing works on coverage for energy harvesting WSNs have only focused on maximizing the coverage probability of targets by using duty cycling [7] or prediction techniques [8]. Briefly, duty cycling algorithms aim to activate sensor nodes in a manner that maximizes events detection. On the other hand, prediction based algorithms calculate the probability of an event occurring at a target's location based on historical information. In comparison to the said works, in this paper, we aim to find the activation time(s) of sensor nodes such that they are afforded recharging opportunities whilst ensuring complete target coverage. We remark that this problem has not been considered in energy harvesting WSNs.

To this end, we propose two algorithms to address the MLC problem in energy harvesting WSNs. We first formulate the Maximum Lifetime Coverage with Energy Harvesting node problem (MLCEH) as a LP with the objective to maximize network lifetime. We show that this LP-based solution doubles network lifetime when compared to similar algorithms developed for finite battery WSNs. However, this LP solution incurs high computational cost due to multiple calls to a LP solver. To this end, we propose Maximum Utility Algorithm (MUA), where utility is the ratio between the number of nodes that are recharging over the number of node with full battery. It has a running time of $\mathcal{O}(|T| \times(|S|+|Z|)$, where $|T|$ is the number of time slots, $|S|$ and $|Z|$ are the number of sensor nodes and targets respectively.

Next, we describe our network model, followed by the problem at hand. We also provide some analysis in Section 3. Section 4 outlines our solutions and its evaluation is documented in Section 5. Section 6 concludes the paper.

\section{Network Model}

We consider a WSN modelled as a sensor-target bipartite graph $(S, Z, E, W)$, where $S$ is the set of sensors, $Z$ is the set of targets, and $E$ is the set of edges connecting a sensor $s_{i} \in S$ to one or more targets in $Z$. Note, we will use $s_{i}$ and $z_{j}$ to index sensors and targets, where $i=1 \ldots|S|$ and $j=1 \ldots|Z|$. Lastly, $w_{i j} \in W$ is an edge weight defined as the residual active time that sensor node $s_{i}$ has for monitoring target $z_{j}$.

Let $E_{i}$ (Joules) be the current energy of sensor node $s_{i}$, which is bounded by the battery capacity $B_{\max }$. In addition, it has a recharging rate of $E_{i}^{r}$ (Joule/s). Also, each sensor consumes $E_{i}^{c}$ (Joule/s) when active. Let $Z\left(s_{i}\right)$ be a function that returns the set of targets covered by sensor $s_{i}$; i.e., sensor $s_{i}$ covers $\left|Z\left(s_{i}\right)\right|$ targets. Conversely, $S\left(z_{j}\right)$ is a function that returns the set of sensors covering target $z_{j}$. Define $C_{t} \subseteq S$ to be the set cover at time slot $t$ that is monitoring at least one target. Let $Z\left(C_{t}\right)$ return the set of targets covered by sensor nodes in set cover $C_{t}$. With a slight abuse of notation, let $W\left(C_{t}\right)$ and $W^{*}\left(C_{t}\right)$ return the set of weights for sensor nodes in and outside of set cover $C_{t}$ respectively. Let $\phi\left(C_{t}, z_{j}\right)$ be a coverage mapping function that returns one if target $z_{j}$ is covered by $C_{t}$, otherwise it returns zero. Also, $E\left(C_{t}\right)$ is an in- 
dicator function that returns one if the residual energy of all sensors in $C_{t}$ is sufficient to provide cover until epoch $\delta_{t+1}$.

Here, an epoch, denoted as $\delta_{t}$, is a time instant defined as one of the following: (i) when a target is not monitored by any sensor node, or (ii) when an in-active sensor node has full battery. In the first case, any developed algorithm needs to activate another set cover to monitor all targets. In the second case, these algorithms need to take into account sensor nodes with a full battery such that they are used to monitor targets whilst affording other nodes recharging opportunities. This means a time slot is the duration between epoch $\delta_{t}$ and $\delta_{t+1}$, where $t=1, \ldots, T$.

\section{Problem Statement}

We now describe the Maximum Lifetime Coverage with Energy Harvesting node (MLCEH) problem. The main objective is to determine the set covers and their corresponding active time such that all targets are monitored continuously. That is, determine the maximum coverage time $t$, where $t \in[0, \infty]$, that satisfies the following constraints: (i) $E\left(C_{t}\right)=1$, and (ii) $\phi\left(C_{t}, Z\right)=1$. Constraint (i) ensures all sensor nodes in cover $C_{t}$ have sufficient energy. Moreover, as per constraint (ii), $C_{t}$ provides complete coverage.

Note that the problem becomes NP-hard, see [1], if the problem is to determine the minimum number of sensor nodes that covers all targets and is equivalent to the minimum set cover problem. Our problem, on the other hand, aims to minimize the activation time of sensor nodes such that all targets are covered, whilst affording them ample time to recharge.

\subsection{Analysis}

Before presenting our algorithms, we present some analysis on continuous coverage. The following are two key definitions used throughout the paper:

Definition 1. Coverage lifetime $(T)$ is the duration in which sensors start monitoring targets until they fail to monitor these targets due to the lack of energy.

Definition 2. Complete target coverage is achieved when all targets are covered by at least one sensor at any time slot t. That is, $\phi\left(C_{t}, Z\right)>0$.

For a given target $z_{j}$ and a target lifetime of $T$, the aggregated energy expenditure of all $\left|S\left(z_{j}\right)\right|$ sensor nodes used to monitor $z_{j}$ must be at least $E_{\text {total }}(T)=T \times E_{i}^{c}$. Let $E_{h}\left(S\left(z_{j}\right)\right)$ be this total amount spent monitoring target $z_{j}$. Then for each target $z_{j} \in Z$, a necessary condition to achieve a lifetime of $T$ is that we must have $E_{h}\left(S\left(z_{j}\right)\right) \geq E_{\text {total }}(T)$.

Note that $E_{h}\left(S\left(z_{j}\right)\right)$ only includes energy spent monitoring a target $z_{j}$. Here, we ignore energy used to power other components such as the microcontroller. If a sensor node is watching multiple targets, then the energy dedicated to $z_{j}$ will be proportional to its time watching target $z_{j}$. Let $x_{i j}$ denote said proportion/fraction of time for sensor $s_{i}$ and corresponding target $z_{j}$. Hence, the total energy used to monitor a target $z_{j}$ is $E_{h}\left(S\left(z_{j}\right)\right)=\sum_{i \in S\left(z_{j}\right)} x_{i j} E_{i}^{c}$. Then, we have the following proposition, 
Proposition 1. Perpetual coverage is achieved when $\sum_{i \in S\left(z_{j}\right)} E_{i}^{r} \geq E_{h}\left(S\left(z_{j}\right)\right)$ for all $j \in Z$.

In other words, if sensor nodes monitoring a target $z_{j}$ is harvesting more energy than they are spending, then said target will be monitored continuously. Note, the above definition does not include recharging efficiency, battery leakage and energy used to power components [3]. If these are included then the amount of harvested energy will have to be significantly higher than the energy used to monitor targets. Following Proposition 1, if we do not have perpetual coverage, then the complete coverage lifetime is governed by the target $z_{j}$ with the smallest aggregated recharged energy and highest monitoring expenditure. Define,

$$
Z_{\text {min }}=\left\{z_{j} \mid \frac{\sum_{i \in S\left(z_{j}\right)} E_{i}^{r}}{E_{h}\left(S\left(z_{j}\right)\right)}<1\right\}
$$

Then the target that fails to be monitored first is,

$$
z_{j}^{*}=\max _{z_{j} \in Z_{\min }}\left[E_{h}\left(S\left(z_{j}\right)\right)-\sum_{i \in S\left(z_{j}\right)} E_{i}^{r}\right]
$$

Hence, target $z_{j}^{*}$ will be watched for a maximum time of

$$
\frac{\sum_{i \in S\left(z_{j}^{*}\right)} E_{i}}{E_{h}\left(S\left(z_{j}^{*}\right)\right)-\sum_{i \in S\left(z_{j}^{*}\right)} E_{i}^{r}}
$$

Note, the denominator of (3) corresponds to the shortfall in harvested energy. The maximum coverage lifetime thus does not exceed the total available energy of sensor nodes monitoring $z_{j}^{*}$.

\section{Solutions}

We now present our solutions to the MLCEH problem. The main difference to prior works is that we consider the recharging capability of sensor nodes. In particular, we need to ensure sensor nodes do not lose any recharging opportunities. This occurs when a sensor node has a full battery, and thereby is unable to store additional energy. Our algorithms run every epoch. Let $x_{i j}^{t}$ denote the time that sensor node $i$ watches target $j$ in time slot $t$. Hence, within a time slot, we have complete target coverage; see Section 2. Moreover, we see that the lifetime of a WSN is simply the epoch $\delta_{T}$ in which an algorithm fails to provide complete coverage.

In the following section, we propose two algorithms to determine the maximum $\delta_{T}$. We first propose a LP based MLCEH algorithm. After that, in Section 4.2, we present MUA, which selects nodes based on their residual energy level.

\subsection{LP based MLCEH algorithm}

The objective of LP-MLCEH is to maximize $\delta_{T}$. To this end, we aim to minimize the energy consumption of each sensor node whilst maintaining complete coverage. We use the following LP to obtain $x_{i j}^{t}$,

$$
\underset{x_{i j}^{t}}{\operatorname{MIN}} \sum_{t=1}^{T} \sum_{i \in S} \sum_{j \in Z} x_{i j}^{t}
$$


Subject to:

$$
\begin{aligned}
& \sum_{i \in S\left(z_{j}\right)} x_{i j}^{t} \geq 1, \quad \forall t=1, \ldots, T, \forall z_{j} \in Z \\
& \sum_{j \in Z\left(s_{i}\right)} x_{i j}^{t} \leq 1, \quad \forall t=1, \ldots, T, \forall s_{i} \in S \\
& B_{\max }-\sum_{t=1}^{T}\left[E_{i}^{r} \sum_{j \in Z\left(S_{i}\right)}\left(1-x_{i j}^{t-1}\right)-E_{i}^{c} x_{i j}^{t}\right] \geq 0
\end{aligned}
$$

Constraint (5) ensures all targets are watched while constraint (6) ensures that each sensor does not exceed its energy in one time slot. Lastly, constraint (7) ensures energy neutral operation; the total energy spent is less than the sum of a node's battery at $t=0$, which we assume to be full, and harvested energy. A suitable $T$ is then determined using binary search.

\subsection{Maximum Utility Algorithm}

Recall that sensor nodes are not able to store or use harvested energy when their battery is at capacity. To this end, we propose MUA to minimize the energy wastage due to lost recharging opportunities. Here, utility is defined as the ratio of the number of nodes that are recharging over the number of nodes with full battery. Every target selects a sensor node with maximal residual energy to be activated at each epoch $\delta_{t}$. Then $\delta_{t+1}$ is defined as the time instant when an active sensor node exhausts its energy or a non-active sensor node has a full battery. Let $L_{t}$ and $R_{t}$ be the minimal operation time of activated nodes and recharging time of non-active nodes respectively. Algorithm 1 shows the pseudo-code of MUA.

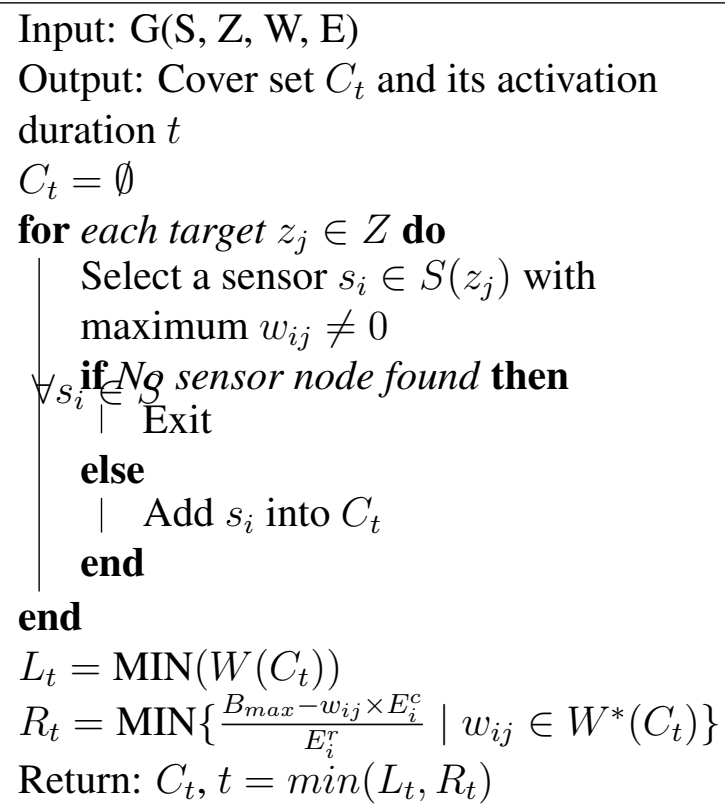

Algorithm 1: Maximum Utility Algorithm

The running time complexity of MUA can be shown to be $\mathcal{O}(|T| \times(|S|+|Z|))$, where $|S|$ and $|Z|$ are the number of sensor nodes and targets respectively and $|T|$ is the number of epochs. In contrast, LP-MLCEH involves the use of binary search to determine $T$ by repeatedly solving an LP containing $T \times(|S|+|Z|)+|S|$ constraints and $|S||Z|$ decision variables. On an Intel Core i7 CPU @ 3.5GHz with 8 G RAM computer, experiments involving 100 randomly generated networks comprising of 20 targets and 20 sensor nodes, the average running time of LP-MLCEH is 11.458 seconds while MUA only needs 0.0627 seconds. 


\section{Evaluation}

We verify our algorithms via simulation using the parameters of the WaspMote [9], which consumes $60 \mathrm{~mW}$ when active and $0.2 \mathrm{~mW}$ when in sleep mode. All sensor nodes are equipped with an Enocean ECS310 solar cell [10]. It has a conversion rate of $10 \%$ and a recharging efficiency of $50 \%$, which is conservative as compared to other technologies [11]. In addition, we use real solar irradiance data retrieved from Southwest Solar Research Park, Phoenix, Arizona, USA [12] on the 16-th of April 2013. Hence, for each sensor node, its recharging rate is a sinusoidal function that peaks at 12 o'clock in every 24 hours period. Other parameter values are as follows: (i) battery size, $1100 \mathrm{~mA}$, (ii) consumption rate, 3.6 Joules/hour, (iii) voltage, $4 \mathrm{~V}$, (iv) solar panel conversion rate, $10 \%$, and (v) recharging efficiency, $50 \%$.

We compare LP-MLCEH and MUA with the Maximum Covers using Mixed Integer Programming (MC-MIP) algorithm proposed in [13], which addresses the MLC problem by dividing sensor nodes into disjoint set covers. In order to run MC-MIP in the energy harvesting case, we first run the MC-MIP algorithm to calculate a network lifetime. We then re-run the algorithm at the end of the computed lifetime. The algorithm re-runs until sensor nodes fail to monitor a target. We also compare LP-MLCEH and MUA with different rules used to select active sensor nodes. These rules include Random, Maximum Energy First (MEF) and Maximum Target First (MTF). For the Random rule, each target is paired with a sensor node randomly. In the MEF and MTF rules, targets select sensor nodes with the most residual energy or cover the most number of targets respectively.

Our results are an average of 100 runs, each with a different topology. In our evaluation, each sensor node has a maximum 76.6 hours worth of energy. However, a network may operate perpetually if it has sufficient number of sensor nodes; see Section 3.1. In our experiments, we define a network lifetime upper bound of 30000 hours, at which time we assume that the network is able to operate perpetually. Recall that, we assume each sensor node is able to communicate with the sink directly and we do not consider energy consumption due to communication. Note, communication cost can be considered by scaling the available energy at each node or by reducing its recharging rate to account for monitoring. In all experiments, we measure network lifetime in the following scenarios: varying number of sensor nodes, number of targets and sensing range.

\subsection{Results}

\subsubsection{Node Density}

We first fix the number of targets to 20 and vary the number of sensor nodes from five to 40 - both sensor nodes and targets are dispersed within a $1000 \times 1000 \mathrm{~m}^{2}$ field. Each sensor node has a uniform sensing range of 500 meters. From Figure 1(a), we see that network lifetime increases rapidly when we add more sensor nodes except when using the Random rule. The reason is that as the number of sensor nodes increases, each sensor node has more opportunities to enter the sleep state, which helps increase harvested energy. For the Random rule, a target may be monitored by multiple, and hence redun- 


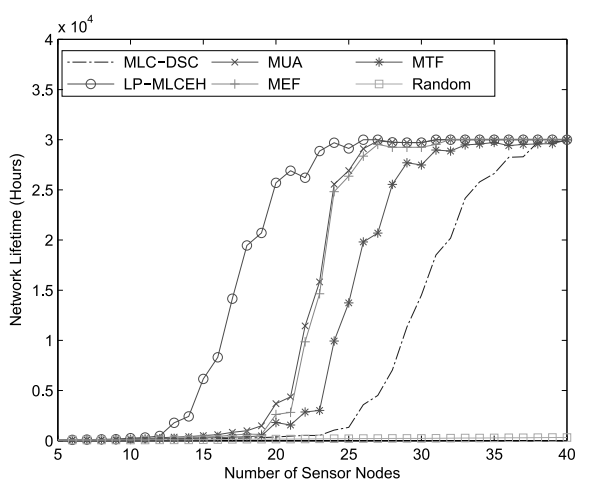

(a) Number of Sensor Node vs. Network Lifetime
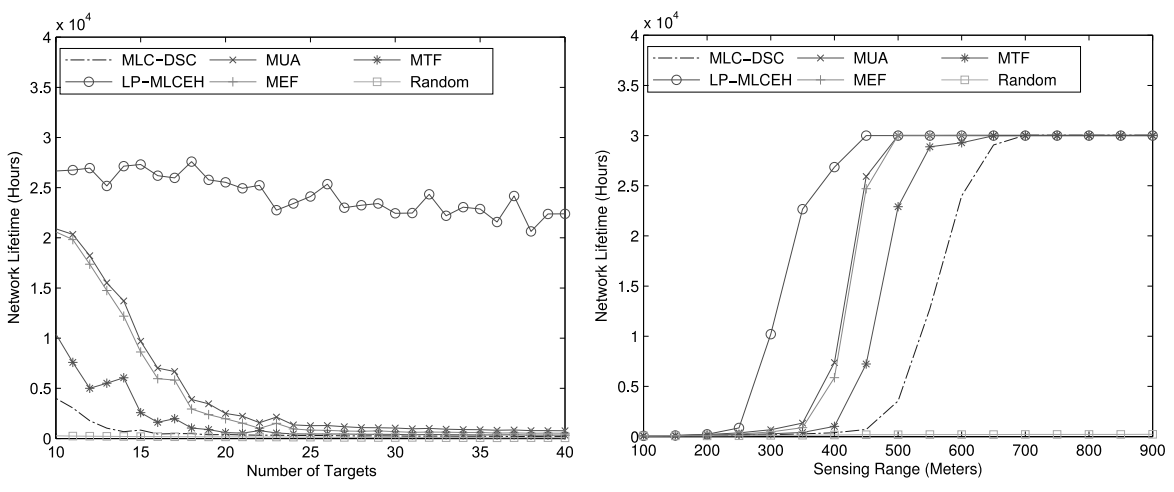

(b) Number of Targets vs. Network Lifetime

(c) Sensing Range vs. Network Lifetime

Figure 1: The impact of number of sensor nodes, targets and sensing range.

dant, sensor nodes. Figure 1(a) also shows LPMLCEH achieves perpetual coverage with minimal number of sensor nodes. On the other hand, to obtain similar performance, MUA requires approximately 10 more sensor nodes, which is $4 / 3$ of the number of nodes required by LPMLCEH. We also see MUA needs one less sensor node than MEF because no energy is wasted due to missed energy harvesting opportunities. Furthermore, MC-MIP requires 15 more, which is 38, sensor nodes than LP-MLCEH to achieve perpetual coverage. Therefore, MC-MIP is not suitable for energy harvesting WSNs.

\subsubsection{Target Density}

We now fix the number of sensor nodes to 20 and vary the number of targets from ten to 50 . Figure 1(b) shows a $20 \%$ reduction in lifetime for LP-MLCEH when the number of targets increases from 10 to 20. However, other algorithms decrease by $80 \%$. MUA has a longer network lifetime than MEF. In particular, the net- work lifetime of LP-MLCEH reduces by $30 \%$ when we continuously add targets while other algorithms recorded more than $90 \%$ reduction.

\subsubsection{Sensing Range}

We now study the effect of sensing range. We disperse 30 sensor nodes and targets within a $1000 \times 1000 \mathrm{~m}^{2}$ field. We then vary sensor nodes' sensing range from 100 to 900 meters. From Figure 1(c), we see that network lifetime increases with different sensing range thresholds for each algorithm except for the Random rule. Upon reaching the threshold, the algorithms rapidly achieve perpetual coverage with 150 meters additional sensing range. The reason is that approximately four more sensor nodes are used to monitor each target. From Figure 1(c), the network lifetime of LP-MLCEH peaked at 450 meters while MUA and MEF require the sensing range to be 500 meters. Moreover, LPMLCEH has twice the network lifetime of MUA when the sensing range increases from 250 to 
400 meters.

\section{Conclusion}

This paper is the first to propose the Maximum Lifetime with Energy Harvesting node (MLCEH) problem, and outlines two novel algorithms. Simulation results show that LPMLCEH doubles the network lifetime as compared to a similar algorithm proposed for finite WSNs. Given that LP-MLCEH involves solving a LP repeatedly at each time epoch, we propose an fast algorithm called MUA that achieves $\frac{3}{4}$ of the network lifetime of LP-MLCEH.

\section{References}

[1] B. Wang, Coverage Control in Sensor Networks, ch. Coverage Lifetime Maximization, pp. 65-95. Springer, 2010.

[2] S. Sudevalayam and P. Kulkarni, "Energy harvesting sensor nodes: Survey and implications," IEEE Communications Surveys \& Tutorials, vol. 13, pp. 443 - 461, Third quarter 2011.

[3] A. Kansal, J. Hsu, S. Zahedi, and M. B. Srivastava, "Power management in energy harvesting sensor networks," ACM Transactions on Embedded Computing Systems (TECS), vol. 6, no. 4, pp. 32-70, 2007.

[4] M. Cardei, M. T. Thai, Y. Li, and W. Wu, "Energy-efficient target coverage in wireless sensor networks," in IEEE INFOCOM, vol. 3, pp. 1976-1984, March 2005.
[5] H. Liu, X. Jia, P.-J. Wan, C.-W. Yi, S. K. Makki, and N. Pissinou, "Maximizing lifetime of sensor surveillance system," IEEE/ACM Transactions on Networking, vol. 15, no. 2, pp. 334-344, 2007.

[6] S. Slijepcevic and M. Potkonjak, "Power efficient organization of wireless sensor networks," in IEEE International Conference on Communications, vol. 2, (Helsinki), pp. 472-476, Jun 2001.

[7] V. Pryyma, D. Turgut, and L. Bölöni, "Active time scheduling for rechargeable sensor networks," Computer Networks, vol. 54, no. 4, pp. 631-640, 2010.

[8] K. Kar and N. Jaggi, "Dynamic node activation in networks of rechargeable sensors," IEEE/ACM Transactions On Networking, vol. 14, no. 1, pp. 15-26, 2006.

[9] "Waspmote datasheet." http: / / www. libelium.com/downloads / documentation/waspmote_ datasheet.pdf.

[10] "Enocean ecs 310." http://www . enocean.com/en/enocean_ modules/ecs-310/.

[11] A. C. Valera, W.-S. Soh, and H.-P. Tan, "Energy-neutral scheduling and forwarding in environmentally-powered wireless sensor networks," Ad Hoc Networks, vol. 11, no. 3, pp. 1202-1220, 2013.

[12] "Nrel: Southwest solar research park." http://www.nrel.gov/midc/ ssrp/. 
[13] M. Cardei and D.-Z. Du, "Improving wireless sensor network lifetime through power aware organization," Wireless Networks, vol. 11, pp. 333-340, May 2005. 